\title{
Searching for Young Type Ia Supernova Remnants in M83
}

\author{
Chuan-Jui $\mathbf{L i}^{1,2}$, You-Hua Chu ${ }^{1,2,3}$ and Po-Sheng $\mathbf{O u}^{1,2}$ \\ ${ }^{1}$ Institute of Astronomy and Astrophysics, Academia Sinica, P.O. Box 23-141, Taipei 10617, \\ Taiwan \\ email: cjli@asiaa.sincia.edu.tw, yhchu@asiaa.sincia.edu.tw \\ ${ }^{2}$ Graduate Institute of Astrophysics, National Taiwan University, Taipei 10617, Taiwan \\ ${ }^{3}$ Department of Astronomy, University of Illinois, 1002 West Green Street, Urbana, Illinois, \\ USA
}

\begin{abstract}
Young Type Ia supernova remnants (SNRs) are characterized by Balmer-dominated optical spectra, well-defined shell morphologies, $>10^{36} \mathrm{ergs} \mathrm{s}^{-1}$ X-ray luminosities, and a lack of massive stars and dense interstellar gas in their vicinity. Applying these characteristics and using archival deep HST and Chandra observations of M83, we search for young Type Ia SNRs in this spiral galaxy. This is a very difficult task!
\end{abstract}

Keywords. ISM: supernova remnants

\section{Introduction}

M83 (NGC 5236), at a distance of $4.61 \mathrm{Mpc}$, is a nearby large grand-design spiral galaxy (see Figure 1). Supernova remnants (SNRs) in M83 have been identified using the criterion $[\mathrm{S} \mathrm{II}] / \mathrm{H} \alpha$ ratio $>0.3$ in Dopita et al. (2010) and Blair et al. (2012). Using a deep, $790 \mathrm{ks}$, Chandra ACIS X-ray observation of M83, Long et al. (2014) compared X-ray point sources with SNR candidates identified from $[\mathrm{S} \mathrm{II}] / \mathrm{H} \alpha$ ratios or nonthermal radio sources, and confirmed 87 SNRs. Using an expanded Hubble Space Telescope (HST) WFC3 survey of M83, in conjunction with the deep Chandra observation of M83, Blair et al. (2014) made a targeted search of young SNRs, resulting in 63 objects.

These surveys have been successful in finding SNRs expanding into a dense medium and exhibiting forbidden lines with high $[\mathrm{S} \mathrm{II}] / \mathrm{H} \alpha$ ratios or young super-luminous SNRs dominated by emission from O-rich SN ejecta. However, young Balmer-dominated Type Ia SNRs have escaped detection because of their lack of forbidden lines. Adopting the characteristics of Type Ia SNRs in the Large Magellanic Cloud (LMC) reported by Ou et al. (2017), we started to search for young Type Ia SNRs in M83.

\section{Search for Young Type Ia SNRs in M83}

The young Type Ia SNRs in the LMC are characterized by (1) Balmer-dominated optical spectra, (2) well-defined shell structure, (3) X-ray luminosities of a few $\times 10^{36}$ $\mathrm{erg} \mathrm{s}^{-1}$, and (4) Population II stellar and interstellar environments.

To search for Balmer-dominated SNRs in M83, we start with the Chandra X-ray sources from Long et al. (2014), and examine the HST H $\alpha$ images for shell structures associated with the X-ray sources. We immediately find it a very difficult task, as our initial search did not turn up any obvious candidates for Type Ia Balmer-dominated SNRs. Only three candidates are marginally identified (shown in Figure 2). 


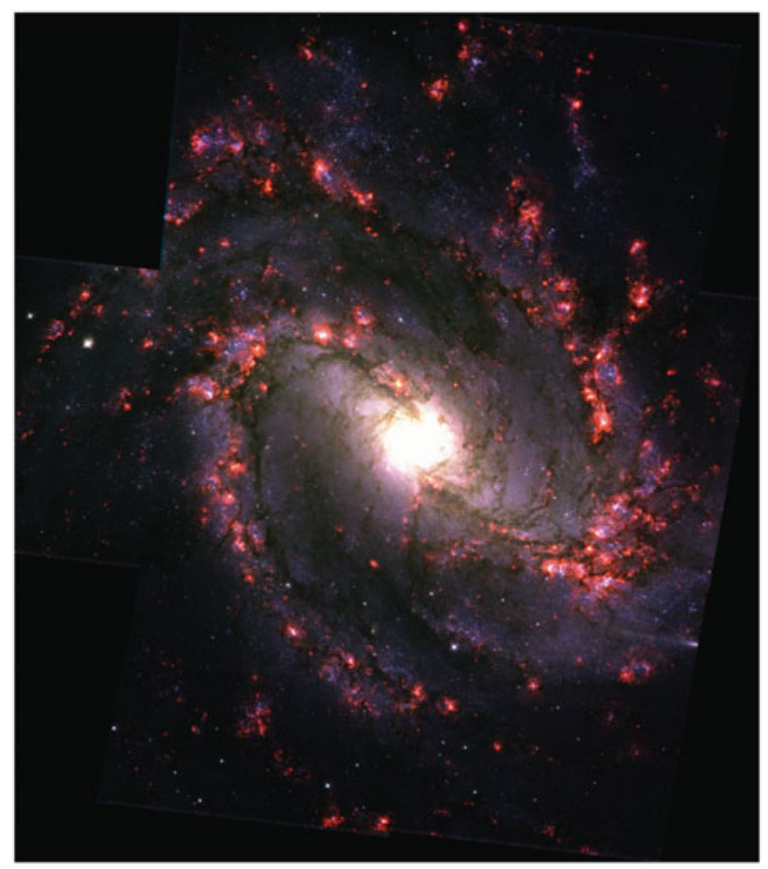

Figure 1. HST image of M83.
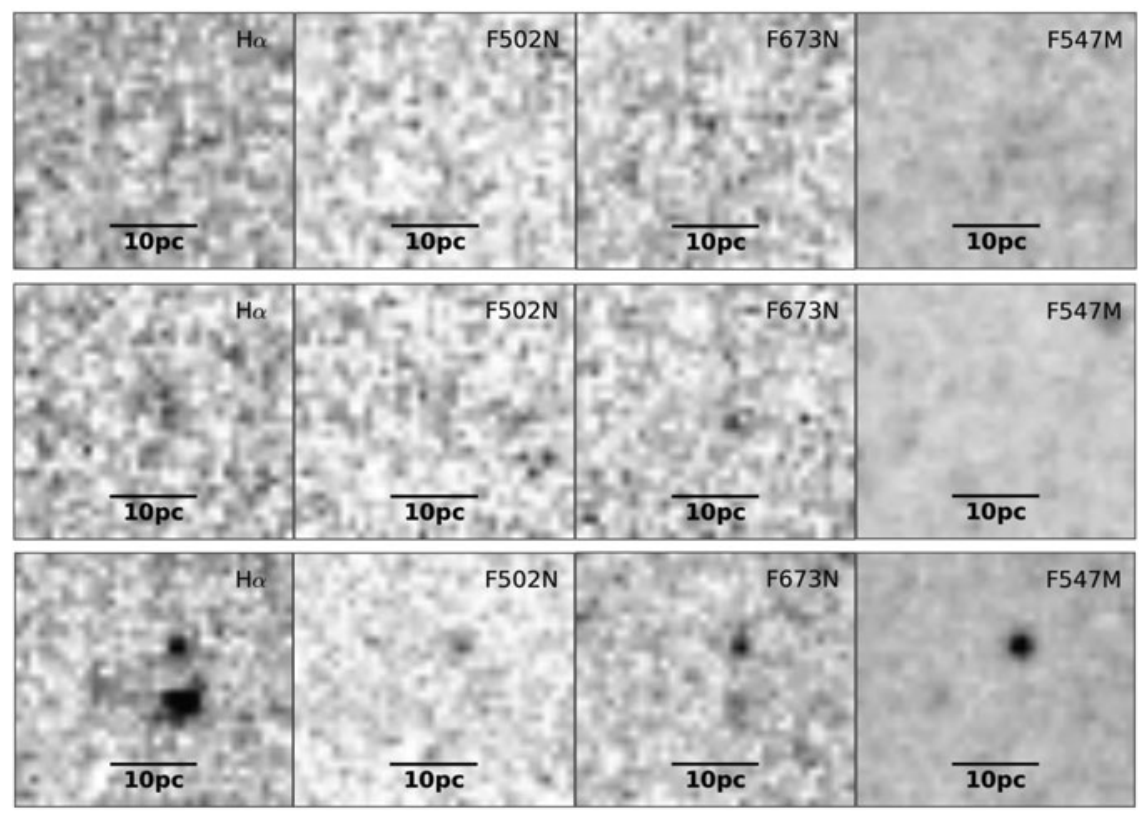

Figure 2. Images of 3 Type Ia SNR candidates (from left to right) showing $\mathrm{H} \alpha(\mathrm{F} 657 \mathrm{~N}),[\mathrm{O}$ III] (F502N), [S II] (F673N), and stellar continuum (F547M).

Are these candidates viable? Why do Balmer-dominated Type Ia SNRs appear so scarce in M83? To answer these questions, we scale their LMC counterparts and estimate their expected surface brightness if located in M83. The SNR 0519-69.0 is a 33" $\times 33$ " Balmer-dominated Type Ia SNR in the LMC at a distance of $50 \mathrm{kpc}$, reported by Smith 


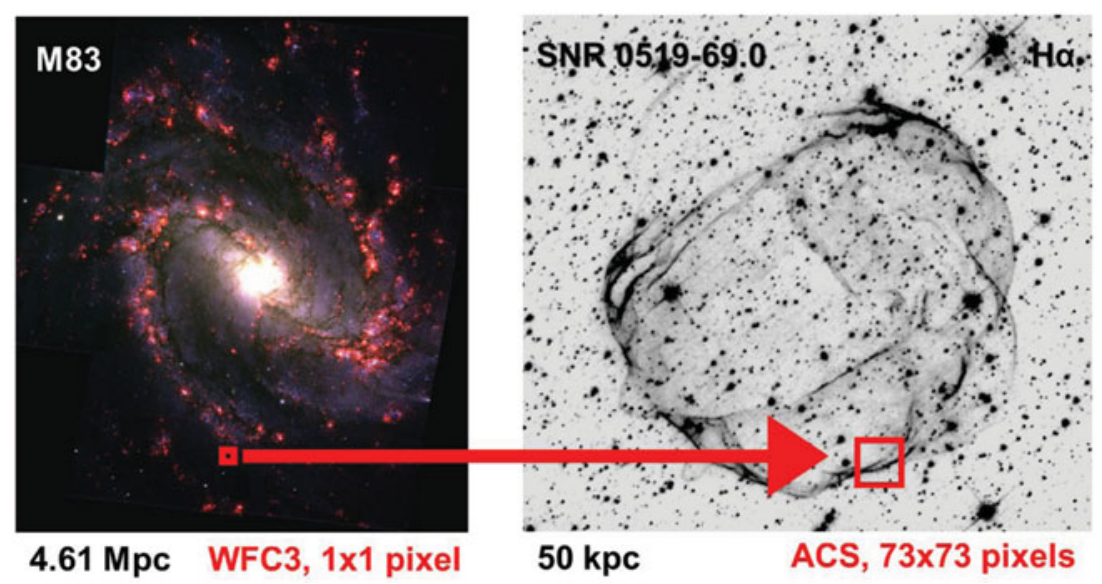

Figure 3. For HST images, a WFC3 pixel $(0.0396$ " $\times 0.0396 ")$ in M83 corresponds to $73 \times$ 73 ACS pixels in the LMC.

et al. (1991). For HST images, a WFC3 pixel $(0.0396 " \times 0.0396 ")$ in M83 corresponds to $73 \times 73$ ACS pixels in the LMC (see Figure 3 ). The Balmer filaments in 0519-69.0 are $\sim 0.3 "$ ( $\sim 6$ pixels $)$ wide; thus, only a small fraction of a $73 \times 73$ area centered on a filament will be filled with emission. The average surface brightness of a $73 \times 73$ area in 0519-69.0 will be its expected surface brightness in M83. We find the average surface brightness of 0519-69.0 to be (4-11) $\times 10^{-16} \mathrm{erg} \mathrm{cm}^{-2} \mathrm{~s}^{-1} \operatorname{arcsec}^{-2}$. The HST H $\alpha$ image of M83 has a background of $\sim 3.4 \times 10^{-16} \mathrm{erg} \mathrm{cm}^{-2} \mathrm{~s}^{-1} \operatorname{arcsec}^{-2}$. Comparisons between these figures indicate that a Balmer-dominated Type Ia SNR in M83 is expected to be detected at generally $\sim 2-3 \sigma$ level and up to $\sim 5 \sigma$ level for the brightest filaments.

The three objects in Figure 2 indeed are detected at $\sim 2-3 \sigma$ levels. Their sizes, smaller than 15pc, are consistent with young SNRs. The lack of dense ionized interstellar gas and massive stars in their vicinity and their X-ray luminosities of a few $\times 10^{36} \mathrm{ergs} / \mathrm{s}$ are consistent with those of young Type Ia SNRs. The detection thresholds of the [S II] or [O III] images are too high to provide meaningful upper limits of these forbidden line emission. We suggest that the three objects in Figure 2 are promising candidates for Balmer-dominated Type Ia SNRs in M83.

\section{Acknowledgements}

This project is supported by MoST grant 104-2112-M001-044-MY3.

\section{References}

Dopita, M. A., Blair, W. P., Long, K. S., et al. 2010, ApJ, 710, 964

Blair, W. P., Winkler, P. F., \& Long, K. S. 2012, ApJS, 203, 8

Blair, W. P., Chandar, R., Dopita, M. A., et al. 2014, ApJ, 788, 55

Long, K. S., Kuntz, K. D., Blair, W. P., et al. 2014, ApJS, 212, 21

Ou, P-.S., Chu, Y.-H., \& Li, C.-J., 2017, poster at this meeting

Smith, R. C., Kirshner, R. P., Blair, W. P., \& Winkler, P. F. 1991, ApJ, 375, 652 